\title{
Mesenchymal Stem Cells in IBD: unMaSCing Their Therapeutic Mechanisms
}

\author{
Franco Scaldaferri ${ }^{1} \cdot$ Stefania Vetrano $^{2,3}$
}

Published online: 24 May 2015

(c) Springer Science+Business Media New York 2015

Given the ethical concerns surrounding the procurement of embryonic stem cells, the study of adult stem cells has become increasingly attractive. In particular, the interest in the growing therapeutic potential of mesenchymal stem cells (MSCs) over the last decade is reflected by the more than 400 clinical trials registered on clinicaltrials.gov and the $>30,000$ scientific publications addressing this subject, which are increasing at a rate of 1200/year (Fig. 1). Despite this extraordinarily rapid growth of knowledge, the effective mechanisms underlying the therapeutic activities of MSCs have not been fully elucidated.

MSCs were described for the first time in 1974 by Friedenstein et al. [1] as adherent, fibroblast-like population of cells present in the bone marrow displaying the ability to differentiate into other mesenchymal cells. Not only can these cells be easily isolated from several easilyaccessible tissues such as adipose tissue and expanded in vitro, but also display self-renewal, multipotent capacity to differentiate into multiple tissue lineages, anti-inflammatory and regenerative activities, and above all were nonimmunogenic, revolutionizing the field of stem cell-based therapy [2].

The ability of MSCs to modulate the immune response by suppressing $\mathrm{T}$ cell proliferation has elevated expectations

Stefania Vetrano

stefania.vetrano@hunimed.eu;

stefania.vetrano@humanitasresearch.it

1 Internal Medicine and Gastroenterology Division, Catholic University of the Sacred Heart, Rome, Italy

2 IBD Center, Humanitas Clinical and Research Center, Rozzano, Italy

3 Department of Biomedical Sciences, Humanitas University, Via Manzoni 113, 20089 Rozzano, Milan, Italy regarding MSC-based therapy in the treatment of inflammatory disease including inflammatory bowel disease (IBD), of which Crohn's disease (CD) and ulcerative colitis (UC) are the major forms. Although their etiology is still unknown, both forms of IBD are the likely result of an abnormal mucosal immune response triggered by specific antigens expressed by luminal bacteria in genetically predisposed individuals [3]. The mainstays of treatment of moderate-severe IBD are immunomodulators and cytokinedirected immune-neutralizing antibodies, which are effective but also require careful monitoring due to potentially severe adverse effects. That MSCs exhibiting immunosuppressive activity without completely inhibiting immune cell function and without myeloablation, in association with their antifibrotic capacity, have fostered their clinical use in IBD patients, in particular in those with fibrosing and fistulising complications. The positive clinical experience reported in the treatment of Crohn's fistulae by local implantation of cells has encouraged initial enthusiasm for the systemic transplantation of MSCs in IBD patients with predominant luminal inflammation [4,5]. Although no adverse effects were reported, confirming the safety of this approach, the benefits are controversial, justifying limited studies in small cohorts of patients. An ongoing phase III, multicentre, doseranging, placebo-controlled study in $>300$ subjects sponsored by Osiris is designed to test the benefits of MSCs in CD patients. Albeit the results of this study will provide important clinical data, it will not address several unresolved questions related to MSC-based therapies, such as their fate post-implantation, the optimum mode of the delivery of cells, including the number of injections, the ideal source of cells, and above all the mechanisms whereby MSCs exert their therapeutic effects. MSCs can modulate the immune response by directly interacting with target cells or by the release of soluble paracrine mediators such as tumor 


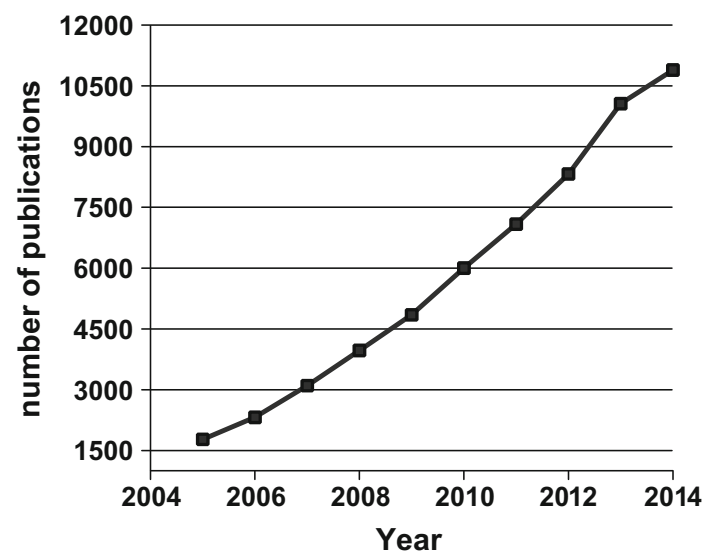

Fig. 1 Number of publications related to MSCs recorded in PubMed per year

necrosis factor $\alpha$-stimulated gene 6 (TSG-6), interleukin-10 (IL-10), transforming growth factor beta (TGF- $\beta$ ), and indoleamine 2,3-dioxygenase (IDO), promoting the expansion of regulatory $\mathrm{T}$ cells while suppressing $\mathrm{T}$ cell activation and proliferation $[6,7]$.

In this issue of Digestive Diseases and Sciences, Tang et al. [8] highlight how granulocyte colony-stimulating factor (G-CSF) promotes the homing of MSCs toward inflamed gut ameliorating colitis. G-CSF, produced in several tissues, mobilizes granulocytes and stem cells including MSC from bone marrow or other tissues into the bloodstream. Moreover, since G-CSF promotes the survival, proliferation, and differentiation of neutrophils, it is used therapeutically to correct neutropenia [9].

Although G-CSF exerts beneficial and therapeutic effects in the treatment of IBD, inducing IL-10-producing T cells, correcting defective neutrophil chemotaxis, and preventing epithelial cell apoptosis, no evidence supports its enhancement of the therapeutic effects of MSCs [10]. Interestingly, Tang et al. observed that combined treatment of MSCs and G-CSF ameliorated colitis by reducing neutrophil infiltration; mucosal NF- $\mathrm{KB}$ activation and systemic concentrations of TNF- $\alpha$ were comparable to either MSC or G-CSF used alone. Furthermore, only the combined treatment elevated serum concentrations of IL-10 and augmented the recruitment of MSCs within inflamed gut. Unexpectedly, MSCs or G-CSF alone non-significantly improved colitis without reducing the amount of neutrophil infiltration. Nevertheless, the authors did not address why and how the combination of MSCs and G-CSF was beneficial, it is plausible to assume that G-CSF recruits circulating MSCs into the gut, promoting epithelial tissue regeneration. Indeed, MSCs mainly engrafted among epithelial cells, suggesting that they helped restore epithelial damage. That elevated serum concentrations of IL-10

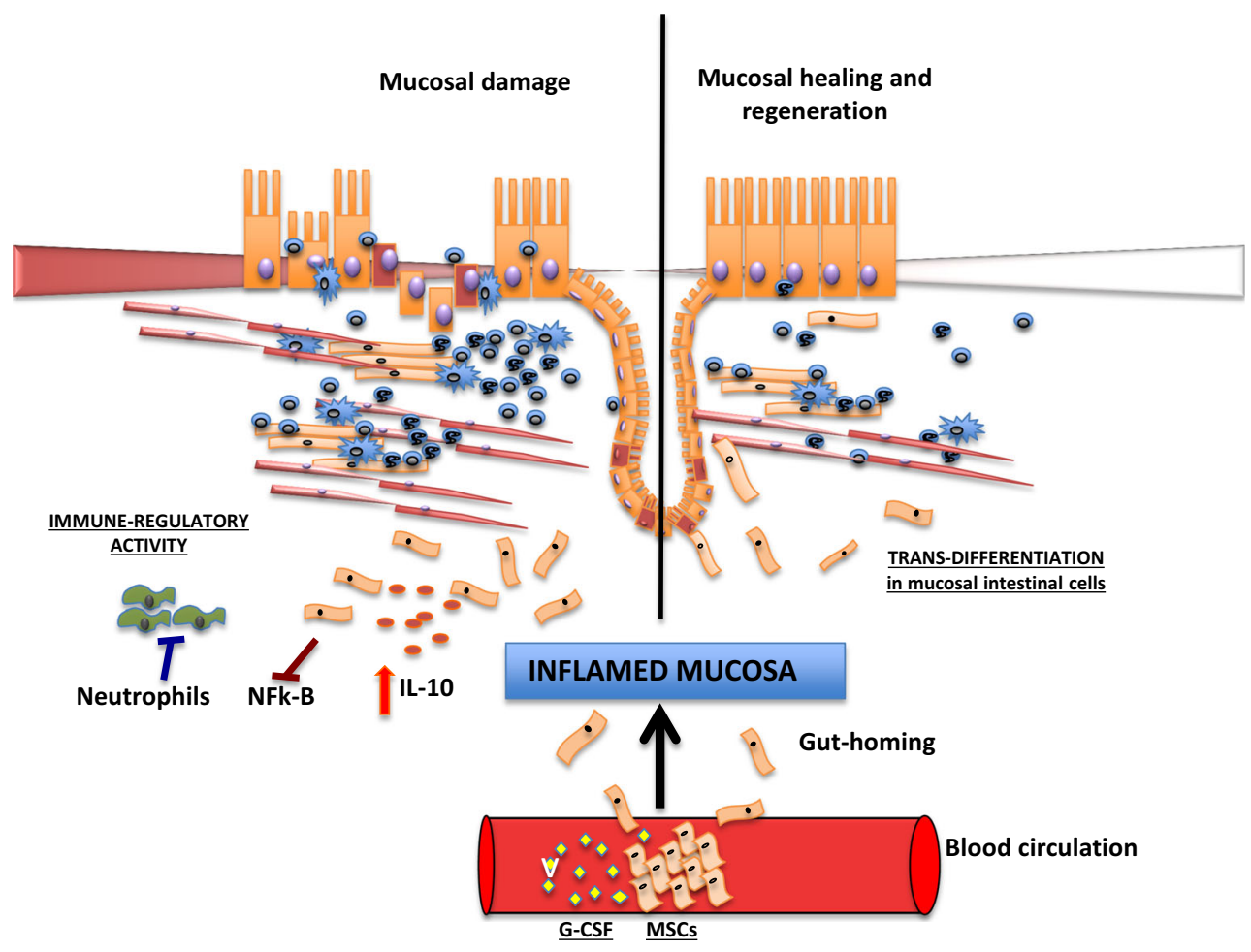

Fig. 2 G-CSF treatment enforces the therapeutic effects of MSCs. The exogenous administration of G-CSF mobilizes the recruitment of MSCs from blood circulation toward the inflamed gut. Once in the gut, MSCs improve colitis by mucosal cell regeneration, and by immune-regulatory activities through release of IL-10 and reduction in neutrophil infiltration and mucosal NF- $\mathrm{KB}$ activation 
observed only after the combined treatment exclude the direct release of IL-10 by G-CSF, but implicate the involvement of gut MSCs, which likely produced IL-10 in response to inflammatory stimuli.

G-CSF, besides mobilizing stem cells, may directly induce immune-regulatory properties in MSCs. The failure of MSCs to reach the gut without the co-treatment with G-CSF confirms that MSCs have a low capacity to home toward inflamed intestine. Although $<1 \%$ of MSCs reached the inflamed gut when administered intraperitoneally (i.p.), most of the cells injected intravenously remained entrapped in the lung without displaying any therapeutic effects. By contrast, i.p. injected MSCs formed lymphoid-like structures exerting anti-inflammatory functions at a distance of the inflamed site [7], posing questions regarding the fate of transplanted MSCs in patients, with attention given to the route of injection and whether experimental colitis models can faithfully mirror clinical disease. Although a conclusive overview of the mode of action MSCs cannot yet be provided, one can argue that MSCs improve colitis by enhancing mucosal cell regeneration and by altering immune-regulatory activities (Fig. 2). Improvements in homing MSCs to the gut by preactivating the cells in vitro before transplantation or by coadministration with mobilizers such as G-CSF may improve the therapeutic effects of MSC-based therapy for the treatment of IBD with predominant luminal inflammation. At this point, it is extremely important to investigate more thoroughly these aspects and to address several unresolved questions related to MSC immunomodulatory and regenerative mechanisms, as well as their ideal mode of delivery, alone or in combination with other factors, before translating MSC-based therapy into clinical practice.
Conflict of interest The authors declare no conflict of interest

\section{References}

1. Friedenstein AJ, Deriglasova UF, Kulagina NN, et al. Precursors for fibroblasts in different populations of hematopoietic cells as detected by the in vitro colony assay method. Exp Hematol. 1974;2:83-92.

2. Chamberlain G, Fox J, Ashton B, Middleton J. Concise review: mesenchymal stem cells: their phenotype, differentiation capacity, immunological features, and potential for homing. Stem Cells. 2007;25:2739-2749.

3. Danese S, Sans M, Fiocchi C. Inflammatory bowel disease: the role of environmental factors. Autoimmun Rev. 2004;3:394-400.

4. Garcia-Olmo D, Garcia-Arranz M, Herreros D, et al. A phase I clinical trial of the treatment of Crohn's fistula by adipose mesenchymal stem cell transplantation. Dis Colon Rectum. 2005;48:1416-1423.

5. Garcia-Olmo D, Herreros D, Pascual I, et al. Expanded adiposederived stem cells for the treatment of complex perianal fistula: a phase II clinical trial. Dis Colon Rectum. 2009;52:79-86.

6. Nauta AJ, Fibbe WE. Immunomodulatory properties of mesenchymal stromal cells. Blood. 2007;110:3499-3506.

7. Sala E, Genua M, Petti L, et al. Mesenchymal stem cells reduce colitis in mice via release of TSG6, independently of their localization to the intestine. Gastroenterology. 2015;. doi:10.1053/ j.gastro.2015.03.013.

8. Tang Y, Chen Y, Wang X, Song G, Shi L, Li Y. Combinatorial intervention with mesenchymal stem cells and granulocyte colony-stimulating factor in a rat model of ulcerative colitis. Dig Dis Sci. (Epub ahead of print). doi:10.1007/s10620-015-3655-3.

9. Fleetwood AJ, Cook AD, Hamilton JA. Functions of granulocytemacrophage colony-stimulating factor. Crit Rev Immunol. 2005;25:405-428.

10. Harbord MW, Marks DJ, Forbes A, et al. Impaired neutrophil chemotaxis in Crohn's disease relates to reduced production of chemokines and can be augmented by granulocyte-colony stimulating factor. Aliment Pharmacol Ther. 2006;24:651-660. 